\title{
Telomerase Activity - a Prognostic Factor in Colorectal Cancer?
}

\author{
Tim H. Brümmendorf \\ Klinik für Onkologie/Hämatologie mit den Sektionen Knochenmarktransplantation und Pneumologie, \\ Universitäts-Klinikum Hamburg-Eppendorf, Germany
}

Telomeres are located at the ends of chromosomes and consist of non-coding TTAGGG repeats and telomere-binding proteins. Telomeres protect the chromosomal ends from degradation, aberrant recombination and end-to-end fusion (capping function). Replication of the 3' end of the lagging strand is incomplete resulting in a loss of roughly 20-200 bp of telomeric DNA with each cell division. Due to this 'end replication problem', telomeres shorten with each round of replication in vitro and in vivo, eventually leading to genetic instability and cellular senescence. These observations led to the proposition of the 'model of a mitotic clock' in which telomere length both reflects the mitotic history of normal somatic cells and predicts their remaining replicative capacity (reviewed in [1]). In contrast to normal somatic cells, germline and cancer cells have been shown to circumvent telomere-mediated cell senescence by maintenance or elongation of telomeres, resulting in an unlimited replicative capacity. The majority of tumor cells investigated so far present with short telomeres and express high levels of telomerase activity, an enzyme capable of adding telomere repeats onto the end of the chromosomes [2]. In humans, this ribonucleoprotein enzyme was shown to contain the 560 bp RNA-matrix (hTR, human telomerase RNA) which is complementary to the human telomere sequence and a reverse transcriptase (hTERT, human telomerase reverse transcriptase) which represents the catalytic subunit of the enzyme. In contrast to the ubiquitous expression of hTR, hTERT expression is mostly restricted to the germline and malignant cells as well as to stem cells. The telomerase-complex also contains a number of associated proteins which are required for the assembly and activity of the enzyme. Although telomerase is the most prevalent mechanism used for maintenance of telomeric DNA, about $10 \%$ of tumors have been described which lack telomerase activity. These cancer cells seem to utilize the so called Alternative Lengthening of Telomeres (ALT) mechanism to circumvent the telomeric checkpoint [3]. It has been postulated that the process of immortalization typically requires the reactivation of telomerase for telomere stabilization and maintenance in tumor cells. Telomere maintenance (+/- telomerase activity) is believed to be an important factor for aging and tumorigenesis as well as for the regulation of the replicative life span of cells [4].

Clinically, telomere length as well as telomerase activity have been demonstrated to be of prognostic value in a variety of human malignancies. Even more importantly, pharmacological inhibition of telomerase activity might represent an attractive new therapeutic target in both solid tumors and hematological malignancies [5]. In addition, telomerase might serve as a promising new (potentially 'pan-tumor') antigen for immunological treatment strategies including vaccination approaches [6].

Telomerase expression can be used to distinguish normal from malignant cells in fine needle aspirates, bronchial washes, and pancreatic juices (reviewed in [7]). The first and clinically most striking evidence supporting the role of telomerase as a prognostic marker in human cancer was published in 1995 by Hiyama et al. in childhood neuroblastoma [8] and has been confirmed by others $[9,10]$. However, in most other solid tumor entities including colon cancer, the published data on the role of telomerase as a clinically useful prognostic marker are controversial. In the current issue of OnKologie, SanzCasla and co-workers report on a prospective study on the prognostic value of telomerase activity for disease outcome in $>100$ patients with colorectal carcinoma [11]. They observe a strinking correlation of telomerase activity with Dukes' stage, tumor site (colon vs. rectum) and most importantly with survival. Furthermore, in a multi-variate analysis, telomerase activity was found to predict both risk of recurrence and death. Although no data on telomere length, telomere-/telomerasebinding proteins or other prognostic parameters (including cytogenetics) are provided and follow-up is too short to draw

\begin{tabular}{ll}
\hline KARGER & (c) 2005 S. Karger GmbH, Freiburg \\
Fax +497614520714 & Accessible online at: \\
$\begin{array}{l}\text { E-mail Information@Karger.de } \\
\text { www.karger.com }\end{array}$ & www.karger.com/onk
\end{tabular}

PD Dr. med. Tim H. Brümmendorf

Klinik für Onkologie, Hämatologie und Pneumologie

Zentrum für Innere Medizin, Universitäts-Klinikum Hamburg-Eppendorf

Martinistraße 52, 20246 Hamburg, Germany

Tel. +49 40 42803-3552, Fax -3563

E-Mail t.bruemmendorf@uke.uni-hamburg.de 
definite conclusions, this study represents another piece of evidence supporting the link between telomerase activity and disease progression in human malignancies.

On the other hand, data on the role of telomere length (for technical reasons) mostly come from studies in hematological malignancies (reviewed in [12]) such as chronic myeloid leukemia (CML) and support models in which the telomere shortening is restricted to the malignant clone (as opposed to normal hematopoiesis), correlated with disease stage and progression [13, 14], prognostic score [15] and response to treatment [16]. However, due to genetic variability in telomere length between individuals, this parameter needs to be indi- vidually related to telomere length of coexisting normal cells in order to further optimize its clinical value. Studies on this issue are currently ongoing.

Taken together, to date, larger series of patients with longterm follow-up are clearly required before telomere length and/or telomerase activity (or hTERT expression) in malignant cells can serve as a prognostic parameter which is valid enough to justify its incorporation into disease-specific treatment algorithms for the individual cancer patient. Moreover, these studies represent the basis for the identification of malignant model disorders that can be used for (early) clinical evaluation of telomerase-directed treatment strategies.

\section{References}

1 Harley CB: Human ageing and telomeres. Ciba Found Symp 1997;211:129-139.

2 Greider CW: Telomerase activation. One step on the road to cancer? Trends Genet 1999;15:109-112.

>3 Bryan TM, Englezou A, la-Pozza L, Dunham MA, Reddel RR: Evidence for an alternative mechanism for maintaining telomere length in human tumors and tumor-derived cell lines. Nat Med 1997;3: 1271-1274.

4 Blasco MA, Hahn WC: Evolving views of telomerase and cancer. Trends Cell Biol 2003;13:289-294.

$\checkmark 5$ Keith WN, Bilsland A, Evans TR, Glasspool RM: Telomerase-directed molecular therapeutics. Expert Rev Mol Med 2002;2002:1-25.

6 Nguyen B, Elmore LW, Holt SE: Telomerase as a target for cancer immunotherapy. Cancer Biol Ther 2003;2:131-136.

7 Hiyama E, Hiyama K: Clinical utility of telomerase in cancer. Oncogene 2002;21:643-649.

8 Hiyama E, Hiyama K, Yokoyama T, Matsuura Y, Piatyszek MA, Shay JW: Correlating telomerase activity levels with human neuroblastoma outcomes. Nat Med 1995;1:249-255.
9 Poremba C, Scheel C, Hero B, Christiansen H, Schaefer KL, Nakayama J, Berthold F, Juergens H, Boecker W, Dockhorn-Dworniczak B: Telomerase activity and telomerase subunits gene expression patterns in neuroblastoma: a molecular and immunohistochemical study establishing prognostic tools for fresh-frozen and paraffin-embedded tissues. J Clin Oncol 2000;18:2582-2592.

10 Krams M, Hero B, Berthold F, Parwaresch R, Harms D, Rudolph P: Full-length telomerase reverse transcriptase messenger RNA is an independent prognostic factor in neuroblastoma. Am J Pathol 2003;162:1019-1026.

11 Sanz-Casla MT, Vidaurreta M, Maestro ML, Fernández C, Jiménez F Arroyo M, Cerdán FJ: Telomerase activity as a prognostic factor in colorectal cancer. Onkologie 2005;28:553-557.

12 Brummendorf TH, Lansdorp PM, Rufer N: Telomere length dynamics in normal and malignant hematopoiesis. J Anti-Aging Med 2000;3:395-407.
3 Brummendorf TH, Holyoake TL, Rufer N, Barnett MJ, Schulzer M, Eaves CJ, Eaves AC, Lansdorp PM: Prognostic implications of differences in telomere length between normal and malignant cells from patients with chronic myeloid leukemia measured by flow cytometry. Blood 2000;95: 1883-1890.

14 Boultwood J, Peniket A, Watkins F, Shepherd P, McGale P, Richards S, Fidler C, Littlewood TJ, Wainscoat JS: Telomere length shortening in chronic myelogenous leukemia is associated with reduced time to accelerated phase. Blood 2000;96: 358-361.

15 Drummond M, Lennard A, Brummendorf T, Holyoake T: Telomere shortening correlates with prognostic score at diagnosis and proceeds rapidly during progression of chronic myeloid leukemia. Leuk Lymphoma 2004;45:1775-1781.

16 Brummendorf TH, Ersoez I, Hartmann U, Bartolovic K, Balabanov S, Wahl A, Paschka P, Kreil S, Lahaye T, Berger U, Gschaidmeier H, Bokemeyer C, Hehlmann R, Dietz K, Lansdorp PM, Kanz L, Hochhaus A: Telomere length in peripheral blood cells reflects response to treatment with Imatinib in patients with chronic myeloid leukemia. Blood 2003;101:375-376. 